\title{
Synthetic Elements for Moving Line Arrays
}

\author{
Dr. Mary H. Johnson, PhD \\ Naval Undersea Warfare Center, Division Newport \\ Code 1522, Bldg 1320 Room 105 \\ 1176 Howell Street, Newport, RI 02842 USA
}

\begin{abstract}
Beamformed line arrays are limited in frequency coverage by both the Nyquist rate imposed by temporal sampling and by the spatial separation of the individual sensors of the array. The spatial limitation, analogous to the Nyquist rate, is imposed by the necessity of adequately sampling the waveform in space in order to distinguish one arrival angle from another. A moving line array effectively samples the space more densely than a stationary array, and, thus, with an adequate temporal sampling rate, meets the sampling criterion for higher frequency coverage than does the stationary array. This paper explores methodology for exploiting array motion to process higher frequency regions at all steered directions in order to use the full frequency capability of the array.
\end{abstract}

\section{INTRODUCTION}

The cost of spatial undersampling is a form of aliasing known as a grating lobe. A single tone produces two or more identical lobes at different look directions. In a stationary array, it can be argued that there is no remedy for the grating lobes because there is no additional information to be exploited. In the case of moving arrays, however, additional spatial sampling results from the array's motion. In particular, as long as the product of the speed of advance of the array and the temporal sampling period is small enough to adequately sample one wavelength of the highest frequency, synthetic elements can be created using a combination of time delay and phase shifting. The proximity of the sensors ensures coherence and we address the basic case of an array with essentially straight line motion. Additional work remains to handle other sensor configurations or changes in array motion.

Our paper describes the application of these concepts to a simulation of a moving line array relative to far-field static sources from a range of azimuths and demonstrates the mitigation of grating lobes using the described technique.

\section{OVERVIEW}

A time domain beamformer for a line array using the delay-and-sum technique calculates the difference between sensors in arrival time of a wavefront in the look direction of interest. The time series received from each sensor is delayed according to this time difference, and then summed to maximize energy received from that look direction. A frequency domain beamformer uses a phase shift or steering term that corresponds to the time delay of the time domain beamformer. Unlike the time delay, the phase shift is frequency dependent and is applied to the Fourier transform coefficients of a batch of time series data from each sensor. Again, the terms are summed. The aliasing that causes grating lobes manifests itself in a frequency domain beamformer by an identical set of steering terms for two or more look directions for the same frequency bin. Increasing the population density of the sensors introduces the intermediate terms that differentiate between these aliased look directions. In this case of a moving array, when information is available due to array motion and a higher temporal sampling rate, it can be used to synthesize additional sampling locations between each physical sensor.

By using the set of time-delayed samples corresponding to this intermediate spacing and applying an additional set of phase shifts to correct for the imposed time delay, we can supplement the physical sensors with an additional set of "synthetic elements" to overcome the spatial aliasing introduced with higher frequencies.

It is important to note that the synthetic element phase shifts depend on the propagated frequency, not the bin frequency, because energy leaks in from neighboring bins, or the observed frequency, which is affected by array motion. The observed frequency will be a function of the relative motion between the array sensor and the source, but the phase shift required to remove the effect of the imposed time delay is a function of the change in phase of the field at a static location. Thus, our algorithm includes the estimation of propagated frequency by removing the effect of array motion. In addition, the use of finite batch sizes in the Fourier transform causes leakage of energy from the mainlobe bin to neighboring bins. We must apply the phase shift to neighboring frequency bins containing significant leakage from the source energy.

\section{BEAMFORMING}

A line array of omnidirectional sensors, moving in a straight line at a constant speed $v$ has $M+1$ sensors spaced $d$ units apart. $f_{D}=c / 2 d$ is the design frequency of the array, where $c$ is the speed of propagation in the medium. The array length is $M d$. For $M$ even, the sensors are numbered from $-M / 2$ to $M / 2$, with sensor 0 at the center of the array. The sampling frequency, $f_{S}$, may be much higher than the design frequency.

Each sensor samples the acoustic field at its current location, collecting samples $x_{m}\left[t_{n}\right]$ from sensor $m$ for time sample $n$. Time domain beamforming uses the delay and sum approach, applying a time delay to each sensor's time samples to align the samples for all $M$ sensors along the 
arrival of a wavefront for each desired look direction. For frequency based beamforming, a vector of steering weights corresponding to the required time shifts for delay and sum beamforming is calculated for each frequency bin. A batch of $N$ samples from each sensor is transformed into a set of $N$ frequency coefficients $X_{m}[k]$ for $k=0$ to $N-1$. The coefficients are phase shifted using a steering vector for each look direction. The steering weights are dependent on sensor number $m$, look direction $\theta$, and bin frequency $f_{k}$ and take the form $\varphi_{m}(\theta, k)=-2 \pi f_{k} m d \cos (\theta) / c$. Beams are formed by summing the weighted coefficients over all sensors:

$$
B[\theta, k, t]=\sum_{m=-M / 2}^{M / 2} X_{m}[k, t] \exp \left(j \varphi_{m}(\theta, k)\right)
$$

for a single bin $k$ at update time $t$.

\section{GRATING LOBES}

The Nyquist theorem dictates that, in order to avoid aliasing in frequency, the sampling rate must be high enough to sample the highest frequency at least twice per cycle. Analogously, in the case of spatial sampling for beamforming, the physical separation of the array sensors must be small enough to sample the spatial waveform at least twice per wavelength.

Consider an array sampling a single tone $s(t)=\exp (j \omega t)$. Grating lobes will appear in the beam response if the sensor separation $d$ exceeds a half-wavelength, $\lambda / 2$, where $\lambda=2 \pi c / \omega$ and $c$ is the propagation speed.

Unlike sidelobes, grating lobes are identical to the mainlobe because they result from the application of an identical steering vector for different look directions. We can define the required conditions for grating lobes to occur by setting these phase shifts equal to each other.

$$
\begin{aligned}
\exp \left(j 2 \pi f m \frac{d}{c} \cos \left(\theta_{a}\right)\right) & =\exp \left(j 2 \pi f m \frac{d}{c} \cos \left(\theta_{b}\right)\right) \\
& =\exp \left(j 2 \pi f m \frac{d}{c} \cos \left(\theta_{b}\right)\right) \exp (j 2 \pi m) \\
& =\exp \left(j 2 \pi m\left(1+f \frac{d}{c} \cos \left(\theta_{b}\right)\right)\right) \\
f \frac{d}{c} \cos \left(\theta_{a}\right) & =1+f \frac{d}{c} \cos \left(\theta_{b}\right) \\
\cos \left(\theta_{a}\right) & -\cos \left(\theta_{b}\right)=\frac{c}{d f}
\end{aligned}
$$

The condition for one grating lobe to occur is dependent on propagation speed $c$, frequency $f$, and sensor separation $d$, as well as the arrival angle of the detected signal.

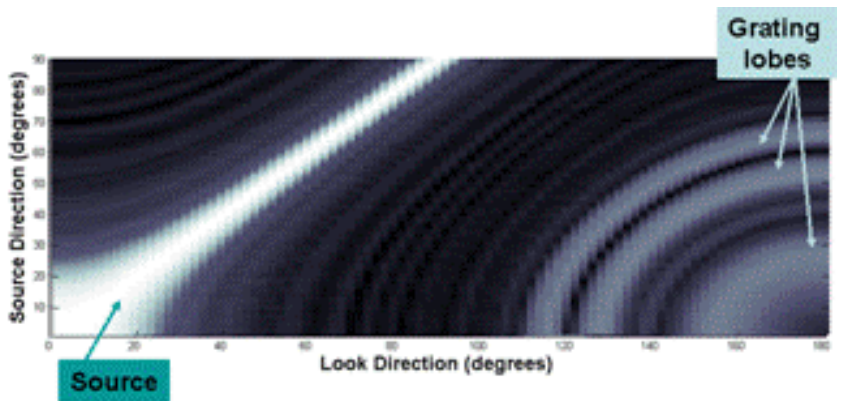

Figure 1: Multi-tone signal with grating lobes from a range of source directions. Each row of this illustration shows the response to the same simulated multi-tone signal arriving from different directions, sweeping from forward to broadside of a line array. The correct main lobe is clearly of larger magnitude because its components cause grating lobes to appear at various look directions based on the frequency content.

From (2), we define a normalized quantity $a$ equal to ratio of the wavelength to the sensor separation. The sum of the two cosine terms must be less than 2 .

$$
\text { Let } a=\frac{c}{d f}=\frac{\lambda}{d} ; \quad a \leq 2
$$

If $a$ is greater than 2, there are no look directions that will satisfy this condition, and there will be no grating lobes. This agrees with our initial assertion that the waveform must be sampled twice in one wavelength. Given the propagation speed and the sensor separation, $a$ will depend on frequency. Grating lobes also depend on the source direction. As the source direction approaches an angle perpendicular to the array, the effective wavelength increases, the cosine term decreases, and a higher frequency is required in order to meet the aliasing condition. That is, as $a$ decreases, there will be aliasing closer to broadside.

Signals with multiple frequency components will produce multiple grating lobes because of this frequency dependence. As stated earlier, the grating lobe is identical to the main lobe for a single frequency component, but for multiple components, the energy will be spread among the grating lobes according to the energy in each component. Fig. 1 illustrates the response to a signal with multiple tones.

\section{DOPPLER}

In an ideal environment where we consider only simple propagation and where source and receiver are motionless, the receiver samples a time-delayed version of the source signal. When the source and/or the receiver is in motion, the received signal is a frequency shifted version of the source signal. To illustrate, define the source signal to be a single tone, $s(t)=\exp (j \omega t)$. For the motionless case, the distance between source and receiver is a constant, $D$, resulting in a fixed time delay, $\tau=D / c$, where $c$ is the speed of propagation in the medium. The fixed time delay equates to a fixed phase shift for the tonal. 


$$
\hat{s}(t, D)=s(t-D / c)=\exp (j \omega t) \exp (-j \omega \tau) .
$$

When the receiver moves relative to the source, the distance, and, therefore, the time delay, become functions of time. If the rate of change is constant, as when the receiver is moving directly towards the source, then the time delay can be expressed as

$$
D(t)=D(0)-v t
$$

where $v$ is the speed of the array and $D(0)-v t$ describes the distance between the source and the receiver at time $t$. The received signal can be expressed in terms of an observed frequency $\omega_{d}$ and a fixed phase shift due to the initial distance $D(0)$. The observed frequency, $\omega_{d}=\omega(1+v / c)$, shows the Doppler effect from the array's motion in the direction of the source.

$$
\begin{aligned}
\hat{s}(t, D(t)) & =s(t-D(t)) \\
& =\exp (j \omega t) \exp \left(-j \omega \frac{D(0)-v t}{c}\right) \\
& =\exp \left(j \omega t\left(1+\frac{v}{c}\right)\right) \exp \left(-j \omega \frac{D(0)}{c}\right) \\
& =\exp \left(j \omega_{d} t\right) \exp \left(-j \omega \frac{D(0)}{c}\right)
\end{aligned}
$$

Now, if another receiver were to follow the same path, but at a time offset of $\tau$, the received signal would have the same observed frequency, with an additional phase shift due to the time offset.

$$
\begin{aligned}
\hat{s}(t-\tau, D(t-\tau)) & =s\left(t-\tau-\frac{D(t-\tau)}{c}\right) \\
& =\exp (j \omega(t-\tau)) \exp \left(-j \omega \frac{D(0)-v(t-\tau)}{c}\right) \\
& =\exp \left(j \omega_{d} t\right) \exp \left(-j \omega \frac{D(0)}{c}\right) \exp \left(-j \omega_{d} \tau\right) \\
& =\hat{s}(t, D(t)) \exp \left(-j \omega_{d} \tau\right) .
\end{aligned}
$$

We need to compensate for the synthetic element time delay by making a phase correction. If we set the time delay to correspond to the time for the array to move a distance, $d,(\tau=d / v)$, then the time delay term can be expanded into two terms, one for the spatial offset and a term for the time delay.

$$
\hat{s}(t-\tau, D(t-\tau))=\hat{s}(t, D(t)) \exp \left(-j \frac{\omega d}{2 c}\right) \exp (-j \omega \tau)
$$

These phase shifts are a function of the propagated frequency, not the observed frequency. The spatial offset term is correct for the synthetic element. The synthetic element phase correction needs to correct for the time delay term, $\exp (-j \omega \tau)$.

The propagated frequency in this far-field example is estimated by compensating for the Doppler shift imposed by array motion:

$$
\omega=\omega_{d} /(1+v / c)
$$

\section{LEAKAGE}

Leakage of energy is a well known phenomenon that inevitably results from the use of a finite batch of data for the Fourier transform. For simplicity, let's look at a signal consisting of a single tonal, $s(t)=\exp (j \omega t)$.

At a distance, $D$, resulting in a time delay, $\tau=D / c$, where $c$ is the propagation speed, we observe a phase shifted version of the original signal,

$$
\hat{s}(t)=s(t-\tau)=\exp (j \omega t) \exp (-j \omega \tau) .
$$

If we sample the signal with sampling frequency $f_{s}$, where sample $n$ is taken at time $t$ such that $n=t f_{s}$, we observe

$$
\hat{s}[n]=\exp \left(j \omega n / f_{s}\right) \exp (-j \omega \tau) .
$$

In order to examine the spectrum, we take $\mathrm{N}$ consecutive samples, and, using a discrete Fourier transform, estimate $N$ frequency coefficients:

$$
\begin{aligned}
\hat{S}[k] & =\sum_{n=0}^{N-1} \exp \left(j \omega \frac{n}{f_{s}}-j \omega \tau\right) \exp \left(-j k 2 \pi \frac{n}{N}\right) \\
& =\exp (-j \omega \tau) \sum_{n=0}^{N-1} \exp \left(j \omega \frac{n}{f_{s}}\right) \exp \left(-j k 2 \pi \frac{n}{N}\right) \\
& =\exp (-j \omega \tau) \sum_{n=0}^{N-1} \exp \left(j \hat{k} 2 \pi \frac{n}{N}\right) \exp \left(-j k 2 \pi \frac{n}{N}\right) \\
\text { for } k & =0: N-1, \quad \text { where } \quad \hat{k}=\frac{\omega N}{2 \pi f_{s}} .
\end{aligned}
$$

We define a number $\hat{k}$ to compare the frequency of the tone with the frequency bin, $k$, of the transform. Now, if $\hat{k}=k$, the terms inside the sum evaluate to 1 and the sum is $N$. If $\hat{k}$ is an integer but not equal to $k$, the sum evaluates to zero. This produces the desired result that the coefficient associated with the tonal frequency is the only non-zero coefficient. However, it is much more likely that $\hat{k}$ is not an integer and the other coefficients are non-zero, their magnitudes being equally spaced points on the sinc-like function shown in [9]. 


$$
\begin{aligned}
\hat{S}[k] & =\exp (-j \omega \tau) \sum_{n=0}^{N-1} \exp \left(j 2 \pi(\hat{k}-k) \frac{n}{N}\right) \\
& =\exp (-j \omega \tau) \sum_{n=0}^{N-1} \exp \left(j \varpi \frac{n}{N}\right) \quad \text { where } \varpi=2 \pi(\hat{k}-k) \\
& =\exp (-j \omega \tau) \frac{1-\exp (j \varpi N)}{1-\exp (j \varpi)} \\
& =\exp (-j \omega \tau) \exp (j \varpi(N-1) / 2) \frac{\sin (\varpi N / 2)}{\sin (\varpi / 2)}
\end{aligned}
$$

The magnitude of this function is traced out in Fig. 2. The coefficient magnitudes are equally spaced points on the curve. This phenomenon is known as leakage because the energy from the closest Fourier transform coefficient "leaks" into the other coefficients. The leakage can be largely confined to a wider main lobe by the use of wellknown windowing functions to taper the edges of the batch.

Spectral leakage is a well documented signal processing artifact, discussed in most signal processing textbooks [2], but the important part of this development for synthetic elements, however, is the recognition that every coefficient $\hat{S}[k]$ is phase shifted by the same term containing the tonal frequency $\omega$, not the individual bin frequency. The synthetic element processing must deal with leakage, first by containing it with a tapering window to keep the bulk of the energy in a smaller neighborhood as shown in Fig. 3. Using an estimate of the propagated frequency, the appropriate phase shift is calculated for application to all bins containing significant leaked energy.
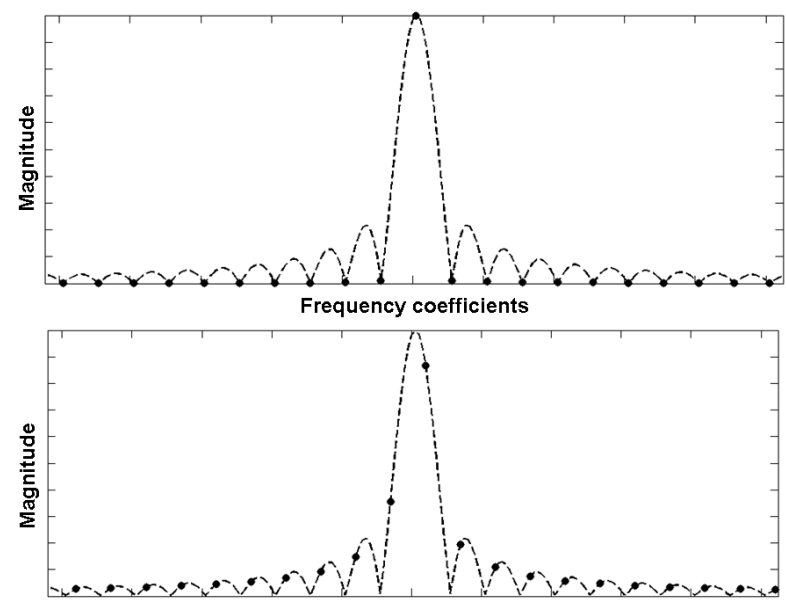

Frequency coefficients

Figure 2 (a) Magnitude of frequency coefficients of Fourier transform for a single tone located at the center of a frequency bin superimposed on the leakage curve. Only one coefficient is non-zero. (b) Magnitude of frequency coefficients of Fourier transform for a single tone located off the center of a frequency bin superimposed on the leakage curve. Significant sidelobes are present. The energy contained in the coefficients of both (a) and (b) are the same, but in (b), energy has leaked into the other bins.
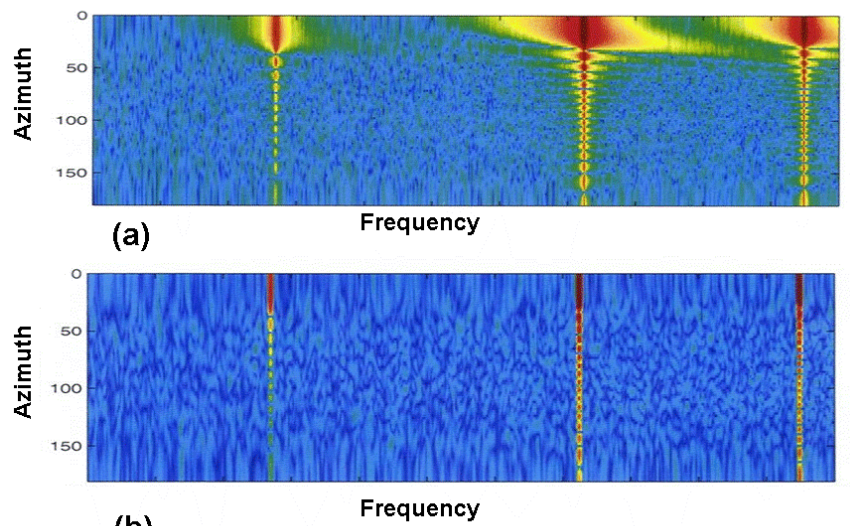

(b)

Figure 3 Leakage examples. (a) A frequency-azimuth plot of beamformed data demonstrating leakage from a multi-tone signal due to the discrete Fourier transform of a non-shaded batch of data. (b) The same signal, with a Blackman window applied prior to the Fourier transform. The signal's mainlobe is about 5 bins wide in frequency, with greatly diminished sidelobes.

\section{BEAMFORMING WITH SYNTHETIC ELEMENTS}

In this case of a moving array, when information is available due to array motion and a higher temporal sampling rate, it can be used to fill in additional synthetic element locations between each physical sensor. We specifically consider a single element between each sensor. Given the array speed $v$, and the sampling rate, it is straightforward to calculate that each sensor will have moved a distance of $d / 2$ in time $d / 2 v$. Phase shifts as a result of this time difference are calculated to be $\exp (-j \omega \tau)$, where the time delay is $\tau=(d / 2 v)$. By using the set of time-delayed samples corresponding to this half-spacing and applying a set of phase shifts to correct for the time delay, we can supplement the physical sensors with an additional set of $M+1$ synthetic elements to mitigate the spatial aliasing introduced with higher frequencies.

This processing consists of three steps. First, loud tonals in the data are detected and a mask is generated for the neighborhood of bins in the main lobe surrounding the peak bin for each tonal. Second, the propagated frequency for each tonal is estimated using the forward motion of the array, the propagation speed, and the peak bin for each tonal. This frequency is used to generate a phase correction for each tonal which is then applied using the mask. Third, the synthetic elements are combined with the real sensors in the frequency beamformer.

The sensor is moving towards the far-field source with a nearly constant speed $v \cos (\theta)$. Starting with a single tone, $s(t)=\exp (j \omega t)$, we sample the signal at a distance $D(t)=D_{0}-v \cos (\theta) t$ from the source.

$$
\begin{aligned}
s(t, f, D(t)) & =\exp (j \omega(t-D(t) / c)) \\
& =\exp \left(j \omega_{d} t\right) \exp \left(-j \omega D_{0} / c\right)
\end{aligned}
$$


where the Doppler frequency is $\omega_{d}=\omega(1+v \cos (\theta) / c)$.

In order to create a synthetic element at a spacing of $d / 2$, we time delay the samples from this sensor by $\tau=d / 2 v$ such that $\hat{t}=t-\tau$.

$$
\begin{aligned}
s(\hat{t}, f, & D(\hat{t})=\exp (j \omega(\hat{t}-D(\hat{t}) / c)) \\
& =\exp \left(j \omega_{d} t\right) \exp \left(-j \omega \frac{D_{0}}{c}-j \omega \tau \frac{v \cos (\theta)}{c}-j \omega \tau\right) \\
& =s(t, f, D(t)) \exp (-j \omega d \cos (\theta) / 2 c) \exp (-j \omega \tau) \\
& =s(t, f, D(\hat{t})) \exp (-j \omega \tau)
\end{aligned}
$$

As described in Section V, in order to keep the phase shift due to the desired spacing and remove the phase shift due to time delay, we separate the total phase shift from the time delay into two parts. The spacing of $\mathrm{d} / 2$ is accounted for by $\exp (j \omega d \cos (\theta) / 2 c)$, while the time delay that we need to compensate for is in the term $\exp (-j \omega \tau)$. Knowing $v$ and $c$, we can calculate the propagating frequency using (6).

For an unknown signal, we will need to first identify the tonal and then apply the phase correction to the energy from this tonal using the Fourier transform coefficients,

$$
\hat{S}[k]=e^{-j \omega \tau} e^{-j \omega \frac{d}{2 c} \cos (\theta)} \sum_{n=0}^{N-1} e^{j 2 \pi(\hat{k}-k) \frac{n}{N}}
$$

As we described in section VI, the finite size of the transform batch will cause leakage of tonal energy into other bins, and that energy will have the phase shift caused by the tonal frequency $\omega$. We must detect the tone in the Fourier coefficients from the sampled signal plus noise $\mathrm{X}[\mathrm{k}]$ and estimate its Doppler frequency, $\omega_{d}$. Then the propagating frequency, $\omega$ can be calculated from (6) and the phase shift to be removed can be calculated as $\exp (-j \omega \tau)$.

Now, we have the necessary information to incorporate the synthetic elements into the beamformer. We start with the physical sensors:

$$
B_{\text {physical }}[\theta, k, t]=\sum_{m=-M / 2}^{M / 2} X_{m}[k, t] \exp \left(j \varphi_{m}(\theta, k)\right)
$$

Where $X_{m}[k, t]$ is the frequency coefficient from bin $\mathrm{k}$ at time update t. we then add the synthetic elements, using the calculated phase correction:

$$
\begin{aligned}
& B_{\text {synthetic }}[\theta, k, t] \\
& =\sum_{m=-M / 2}^{M / 2} X_{m}[k, t-\tau] \exp \left(j \varphi_{m+\frac{1}{2}}(\theta, k)\right) \exp (j \omega \tau) \\
& =\sum_{m=-M / 2}^{M / 2} X_{m+\frac{1}{2}}[k, t] \exp \left(j \varphi_{m+\frac{1}{2}}(\theta, k)\right)
\end{aligned}
$$

Where

$$
\begin{aligned}
\varphi_{m+\frac{1}{2}}(\theta, k) & =\frac{\omega_{k}\left(m+\frac{1}{2}\right) d \cos (\theta)}{c} \\
& =\frac{\omega_{k} m d \cos (\theta)}{c}+\frac{\omega_{k} d \cos (\theta)}{2 c}
\end{aligned}
$$

A simple change of variables shows that the two combine to make the single, more heavily populated array.

$$
\begin{aligned}
B_{\text {combined }}[\theta, k, t] & =B_{\text {physical }}[\theta, k, t]+B_{\text {synthetic }}[\theta, k, t] \\
& =\sum_{m=-M / 2}^{M / 2} X_{m}[k, t] \exp \left(j \varphi_{m}(\theta, k)\right) \\
& +\sum_{m=-M / 2}^{M / 2} X_{m+\frac{1}{2}}[k, t] \exp \left(j \varphi_{m+\frac{1}{2}}(\theta, k)\right) \\
& =\sum_{\hat{m}=-M}^{M} X_{\hat{m}}[k, t] \exp \left(j \varphi_{\hat{m}}(\theta, k)\right)
\end{aligned}
$$

\section{CONCLUSIONS}

We have formulated the concept of using the motion of a line array with a higher sampling rate to enable processing a higher frequency range than is indicated by the physical sensor separation distance. Synthetic elements are formed by using time-delayed samples from the physical sensors. The time delay is calculated to achieve the desired sensor spacing and must be compensated for with a frequency specific phase shift. We have discussed the methods for calculating and applying the correct phase shift.

\section{FUTURE WORK}

Additional work remains to extend this technique to broader signals which are not simple tones and which may overlap in frequency, especially given leakage. We will explore the effects of discrete sampling and of inaccurate Doppler correction. Finally, this work is appropriate for extension to compensating for failed sensors in a line array. 


\section{ACKNOWLEDGMENT}

The author would like to thank Dr. Robert LaTourette and Mr. Steve Greineder for their encouragement in pursuing this concept, as well as Dr. Joseph Dibiase, Dr. Tod Luginbuhl, and Ms. Kelly Ouellette for their many helpful comments.
REFERENCES

[1] M. H. Johnson and R. LaTourette, "Processing Towed Array Data Above the Spatial Design Frequency", NDIA 2005 Joint Undersea Warfare Technology Spring Conference, 30 March 2005..

[2] R. Cristi, Modern Digital Signal Processing, Brooks/Cole Pub Co, 2003. 